Research Paper

\title{
Risk factors for detection of SARS-CoV-2 in healthcare workers during April 2020 in a UK hospital testing programme
}

\author{
John S Leeds ${ }^{\mathrm{a}}$, Veena Raviprakash ${ }^{\mathrm{b}}$, Thomas Jacques $^{\mathrm{c}}$, Noel Scanlon ${ }^{\mathrm{d}}$, Jeremy Cundall ${ }^{\mathrm{e}}$, \\ Clare M Leeds $\mathrm{s}^{\mathrm{f}, *}$ \\ ${ }^{a}$ Population Health Sciences Institute, Newcastle University, United Kingdom \\ ${ }^{\mathrm{b}}$ Department of Microbiology, County Durham and Darlington NHS Foundation Trust, United Kingdom \\ ${ }^{c}$ Department of Infection Prevention \&' Control, County Durham and Darlington NHS Foundation Trust, United Kingdom \\ ${ }^{\mathrm{d}}$ Executive Director of Nursing, County Durham and Darlington NHS Foundation Trust, United Kingdom \\ e Medical Director, County Durham and Darlington NHS Foundation Trust, United Kingdom \\ ${ }^{\mathrm{f}}$ Department of Occupational Health and Wellbeing, Bishop Auckland Hospital, County Durham and Darlington NHS Foundation Trust, Cockton Hill Road, Bishop \\ Auckland, County Durham DL14 6AD, United Kingdom
}

\section{A R T I C L E I N F O}

\section{Article History:}

Received 14 May 2020

Revised 29 July 2020

Accepted 31 July 2020

Available online 17 August 2020

\section{Keywords:}

COVID-19

SARS-CoV-2

Healthcare workers

Diagnostic test

\begin{abstract}
A B S T R A C T
Background: Healthcare workers (HCW) are a crucial part of the workforce but are also at potentially at increased risk of infection from SARS-CoV-2. Emerging evidence has suggested specific groups of HCW are at further increased risk particularly those from Black, Asian and Minority Ethnic (BAME) groups. Previous reports have not examined risk factors associated with contracting the virus and were reported prior to the pandemic peak in the UK.

Methods: Staff testing in our facility commenced on the 1st April and all individuals were entered into a database. Repeat testing was used for the first 3 weeks for those initially testing negative. Demographics including age, sex, occupation and ethnicity were recorded. Occupation was divided into acute frontline (e.g. ITU), frontline, clinical support staff and non-clinical staff. Final testing status was analysed using univariate and multivariable analysis to determine independent associations with age, sex, occupation and ethnicity.

Findings: 991 individuals (mean age 42.6 years, 145 males) were tested over a 4 week period and overall $440 /$ 991 (43.4\%) tested positive for SARS-CoV-2. SARS-CoV-2 RNA detection rates were significantly higher in the first week of testing (59.0\%) compared to week 2 (odds ratio 0.59), week 3 (odds ratio 0.32) and week 4 (odds ratio 0.23 )(all $p<0.001$ ). Multivariable analysis showed no increased risk SARS-CoV-2 RNA detection with age (odds ratio $1.01,95 \%$ confidence interval $0.99-1.03, p=0.22$ ), male sex (OR 1.03, 95\% CI $0.59-1.79$, $p=0.92$ ), acute frontline work (OR 0.79, 95\% CI $0.53-1.17, p=0.23$ ) or BAME status (OR 1.08, 95\% CI 0.56 2.07, $p=0.84)$.

Interpretation: A robust healthcare worker testing strategy is a crucial component of managing the workforce during the SARS-CoV-2 pandemic. No specific variables were identified that altered the risk of SARS-CoV-2 RNA detection including age, sex, occupation and ethnicity.
\end{abstract}

(C) 2020 The Authors. Published by Elsevier Ltd. This is an open access article under the CC BY-NC-ND license. (http://creativecommons.org/licenses/by-nc-nd/4.0/)

\section{Introduction}

The severe acute respiratory syndrome coronavirus 2 (SARS-CoV2) pandemic has been a major challenge for healthcare systems throughout the world. As of 3rd May 2020, Public Health England (PHE) has reported $>186,000$ cases and $>28,000$ deaths in the UK [1]. One of the key strategies for reducing the spread of the virus has been testing of healthcare workers as it was clear that there was a

\footnotetext{
* Corresponding author.

E-mail address: clare.leeds@nhs.net (C.M. Leeds).
}

significant burden in Hubei province [2,3]. Reports from 2 different centres in the UK have shown some advantages of this strategy in the early phase of the pandemic $[4,5]$. Both of these reports analysed the role of staff testing during March 2020 prior to the peak of the pandemic in the UK and both had detection rates of $18 \%$ and $14 \%$ respectively $[4,5]$. Both reports supported return to work for healthcare workers who tested negative and symptoms had subsided. One report provided evidence that nosocomial transmission of SARS-CoV2 is unusual as the detection rate was not significantly different in frontline healthcare workers compared to non-clinical staff [5]. This has also been supported by a small study in a primary care setting 6] Both of these reports analysed the period prior to country wide 


\section{Research in Context}

\section{Evidence Before This Study}

Healthcare workers are potentially at increased risk of contracting SARS-CoV-2 and there is concern that this may be higher still for some individuals such as those in the BAME community. Previous reports have examined risk factors associated with clinical course once diagnosed but not variables associated with contracting the virus.

\section{Added Value of This Study}

Age, sex, occupation and ethnicity are not associated with increased risk of contracting SARS-CoV-2.

\section{Implications of all the Available Evidence}

Healthcare workers with appropriate personal protective equipment and isolation protocols are not at increased risk of contracting SARS-CoV-2 in the work environment. Age, sex, occupation and ethnicity do not modify the risk of contracting SARS-CoV-2 but clearly have been shown to alter the clinical course once the disease is acquired. This data will help to inform policy when risk assessing healthcare workers during the coronavirus pandemic.

restrictions implemented by the government, each used a single testing strategy and did not examine if there were any features associated with increased risk of testing positive for SARS-CoV-2. There have been significant concerns that individuals from Black, Asian and Minority Ethnic (BAME) groups are at a higher risk from SARS-CoV-2 [7-9]. It is unclear whether current increased incidence in mortality within this group is due to increased incidence of infection, increased co-morbidity or other extrinsic factors [8,9]. We report the results of the staff testing programme for April 2020 in a large acute NHS Trust in the UK and examine the characteristics of affected healthcare workers including ethnicity.

\section{Methods}

County Durham and Darlington NHS Foundation Trust (CDDFT) employs circa 7500 (of which 750 are medical), including temporary staff throughout 2 main hospital sites, 6 community hospitals and additional community services. Between these sites, there are 950 hospital beds including 22 for intensive care. During the pandemic this was expanded to 1250 hospital beds with 60 for intensive care. The Trust delivers acute and chronic medical and surgical services and is similar to other institutions within the UK [4,5]. On the 1st April, testing was commenced for staff with symptoms suggestive of SARS-CoV-2 coordinated by the Occupational Health and Wellbeing department. Strict criteria were applied such that only staff members with fever and/or persistent cough within 5 days of onset of symptoms were eligible for testing. This criteria was in line with the self isolation guidance provided by Public Health England. Staff had already been following PHE guidance and were self isolating for 7 days if they had experienced high temperature or persistent cough. Occupational health staff carried out a telephone screening assessment and then arranged booked appointments into one of the onsite testing pods. All swabs were taken by fully trained and dedicated staff using standardised methods from the oropharynx alone or oropharynx and nasopharynx. Swabs were securely sealed and transported to the regional testing laboratory where the samples were assayed for SARS-CoV-2 RT-PCR (RdRp assay; Public Health England) which is the antigen (RNA testing). Results were received by the Occupational Health and Wellbeing department as either "detected" or "not detected". During the period 1st April to 20th April, those receiving a "not detected" result were contacted and a second swab arranged to account for potential false negative results. On receiving final results, staff were sent an email with clear instructions regarding continuing self isolation or return to work . Repeat testing yielded a further 16 SARS - CoV-2 RNA detected results out of 384 repeated tests (4.2\%), therefore this process was discontinued with the assumption that our methods were yielding lower false negatives than expected and that as $95.8 \%$ of those tested double negative that we could switch to the method used by surrounding trusts. Single testing commenced from 21st April.

Staff were therefore designated with a final status of "detected" or "not detected". Staff with a final status of "not detected" were able to return to work if their symptoms had resolved or if the only

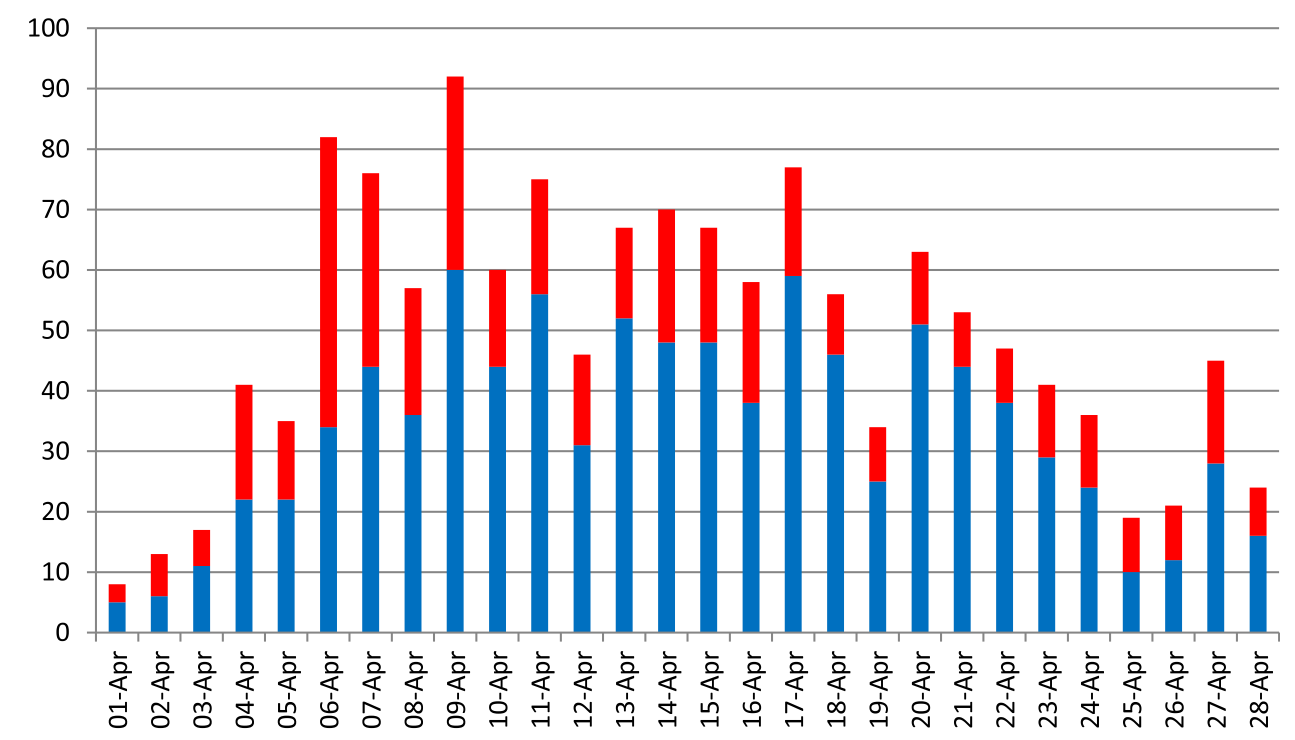

Key: Red $=$ detected. Blue $=$ not detected. 
remaining symptom was cough. Staff who tested positive were told to self isolate until at least day 8 , return to work was then appropriate once symptoms had resolved.

A prospective database was used to track staff who had undergone SARS-CoV-2 testing and included age, sex, ethnicity and occupation. Occupational roles were divided into several categories including nursing/medical, allied healthcare professional, clinical support staff and non-clinical staff. Occupations were divided into 4 groups; acute frontline services (e.g. accident and emergency, intensive care, acute admissions), patient facing services (e.g. medical wards, surgical wards), non-patient facing but possible higher risk (e.g. domestic, laboratory staff) and non-clinical staff. Ethnicity was coded as per the 2001 census data.

\subsection{Data analysis}

Data was analysed using MedCalc Statistical Software version 19.2.1 (MedCalc Software Ltd, Ostend, Belgium; https://www.medcalc.org; 2020). Trends in test results were displayed graphically using final status of each individual from first day of testing. Differences in age, sex, ethnicity and occupation were analysed using proportional analysis with either the chi squared test or Fishers exact test. Occupation was also analysed as per Hunter [5] and colleagues to allow comparison at a different phase of the pandemic. Multiple logistic regression analysis was also performed to determine whether any independent variables were associated with SARS-CoV-2 RNA detection. To assess for interaction between variables the analysis was repeated using different combinations of interaction variables. The combinations used were sex*occupation, sex*BAME and BAME*occupation.

\subsection{Ethical considerations}

This report is a description of the standard clinical care provided by the Occupational Health and Wellbeing department at CDDFT and was registered with the clinical effectiveness department. This study adhered to the Strengthening the Reporting of Observational Studies in Epidemiology (STROBE) guidelines for reporting observational studies.

\subsection{Role of the funding source}

This study was not funded.

\section{Results}

Over the period 1st April 2020 to 28th April 2020, 1378 tests for SARS-CoV-2 were performed in 991 healthcare workers at CDDFT (145 males, mean age 42.6 years). 387 staff underwent repeat testing due to non detection of SARS - CoV2 RNA during the repeat testing period. Of this group, SARS - CoV-2 RNA was detected in additional 16 individuals on the second test. Therefore, overall incidence of SARS - CoV2 RNA detection was 440/991 (43.4\% 95\% confidence interval $41.3 \%$ - $47.5 \%$ ). 551 staff members were eligible for return to work if symptoms allowed. To our knowledge, a very small number of those in whom SARS-CoV-2 RNA was detected were hospitalised.

Table 1

SARS-CoV-2 positive testing rates per week.

\begin{tabular}{lllll}
\hline & Number of tests & Detected (\%) & $\begin{array}{l}\text { Odds ratio }(95 \% \\
\text { confidence interval) }\end{array}$ & $\mathrm{p}$ \\
\hline Week 1 & 222 & $131(59.0 \%)$ & Reference & - \\
Week 2 & 301 & $138(45.8 \%)$ & $0.59(0.41-0.84)$ & 0.0029 \\
Week 3 & 258 & $95(36.8 \%)$ & $0.32(0.22-0.46)$ & $<0.0001$ \\
Week 4 & 210 & $76(36.2 \%)$ & $0.23(0.16-0.34)$ & $<0.0001$ \\
\hline
\end{tabular}

Table 2

Demographic features and SARS-CoV-2 incidence in healthcare workers.

\begin{tabular}{|c|c|c|c|}
\hline Variable & $\mathrm{N}$ & Detected (\%) & $\mathrm{p}$ \\
\hline \multicolumn{4}{|l|}{ Age band } \\
\hline$\leq 24$ & 62 & $31(50 \%)$ & 0.10 \\
\hline $25-34$ & 238 & $100(42.0 \%)$ & \\
\hline $35-44$ & 218 & $85(39.0 \%)$ & \\
\hline $45-54$ & 296 & $137(46.3 \%)$ & \\
\hline $55-64$ & 162 & $83(51.2 \%)$ & \\
\hline$\geq 65$ & 15 & $4(26.7 \%)$ & \\
\hline \multicolumn{4}{|l|}{ Sex } \\
\hline Male & 145 & $63(43.4 \%)$ & 0.86 \\
\hline Female & 846 & $377(44.6 \%)$ & \\
\hline \multicolumn{4}{|l|}{ Ethnicity } \\
\hline White & 572 & $244(42.7 \%)$ & 0.37 \\
\hline Indian subcontinent & 22 & $10(45.5 \%)$ & \\
\hline Chinese Asian & 4 & $4(100 \%)$ & \\
\hline Other Asian & 9 & $3(33.3 \%)$ & \\
\hline Mid East & 2 & $1(50 \%)$ & \\
\hline African & 5 & $1(20.0 \%)$ & \\
\hline Other ethnic group & 7 & $2(28.6 \%)$ & \\
\hline Not stated/declined & 370 & $175(47.3 \%)$ & \\
\hline \multicolumn{4}{|l|}{ Occupational role } \\
\hline Nursing/medical & 584 & $282(48.3 \%)$ & 0.19 \\
\hline Allied health professional & 73 & $34(46.6 \%)$ & \\
\hline Clinical support staff & 29 & $8(27.6 \%)$ & \\
\hline Non clinical & 52 & $24(46.2 \%)$ & \\
\hline
\end{tabular}

Fig. 1 shows the daily breakdown of staff testing results with a clear rapid increase in tests performed per day peaking in week 2 and then slowly reducing by week 4 . SARS - CoV-2 RNA detection rates were significantly higher in the first week of testing (59.0\%) compared to subsequent weeks and is shown in table 1. SARS -CoV-2 RNA detection was higher in the repeat testing period (364/781 vs $76 / 210$, $p=0.008$ ) however this is likely due to the increased incidence in the tested population in the first three weeks. There was no difference in the SARS - CoV-2 RNA detection rate when comparing week 3 to week $4(95 / 258$ vs $76 / 210, p=0.88)$.

Table 2 shows the different characteristics of the cohort with incidence of SARS - CoV-2 RNA detection for each demographic. Data was available for occupational role for 738 individuals (74.5\%). There was no significant difference in SARS - CoV-2 RNA detection rates when comparing different age bands $(p=0.10)$ or sex $(p=0.86)$. Although the incidence of SARS - CoV-2 RNA detection was lower in clinical support staff (27.6\%) compared to the other groups this was not statistically significantly different $(p=0.19)$. Those who were judged to be in acute frontline services did not have significantly different incidence of SARS - CoV-2 RNA detection compared to the other groups $(107 / 227$ vs. $243 / 517, p=0.97)$. Using the same occupational groups as Hunter and colleagues [5] there were no statistically significant differences in SARS - CoV-2 RNA detection between the groups (group $148.1 \%$, group $236.0 \%$, group $342.6 \%, p=0.21$ ). The incidence of SARS-CoV2 RNA detection in the group with missing occupational role was 90/253 (35.6\%).

Regarding ethnicity, there were 658 (66.4\%) individuals with a recorded ethnicity of which 51 (7.8\%) were BAME codes. In February

Table 3

Results of multivariable analysis for SARS-CoV-2 testing.

\begin{tabular}{llll}
\hline Variable & Adjusted odds ratio & $95 \%$ confidence interval & $\mathrm{p}$ \\
\hline Age & 1.01 & $0.99-1.03$ & 0.22 \\
Male sex & 1.03 & $0.59-1.79$ & 0.92 \\
Acute frontline worker & 0.79 & $0.53-1.17$ & 0.23 \\
BAME status & 1.08 & $0.56-2.04$ & 0.84 \\
\hline
\end{tabular}

BAME = Black Asian and Minority Ethnic. 
2020, the ethnicity data was collated for the 6577 permanent staff within the CDDFT of which 6.4\% (417 staff) were registered as BAME. The proportion of BAME individuals in the Trust is not significantly different from this sample $(p=0.19)$. There was no statistically significant difference in incidence of SARS - CoV-2 RNA detection between BAME and non-BAME groups ( $45.1 \%$ vs. $45.3 \%$, odds ratio $0.99,95 \% \mathrm{CI}$ $0.56-1.76, p=1.00$ ). However, of the 51 individuals with BAME status $22 / 51$ (43.1\%) were doctors compared to $25 / 572(4.4 \%)$ in the non-BAME group. Therefore, individuals with BAME status were significantly more likely to be doctors compared to their non-BAME colleagues (odds ratio 16.6, 95\% confidence interval 8.4 - 32.9, $p<0.0001$ ).

Multivariable analysis showed no independent variables were associated with testing positive for SARS-CoV-2 and the results are shown in table 3. Interaction analysis showed no significant interaction between sex and occupation $(p=0.75)$, sex and BAME status $(p=0.28)$ or BAME status and occupation $(p=0.14)$.

\section{Discussion}

This report reveals several important insights into the SARS-CoV2 pandemic in England. There was a very high incidence of testing positive for SARS-CoV2 antigen particularly in the first week of testing. We believe this is likely to be multi-factorial and related to more stringent testing criteria at that time which included only persistent cough and/or fever. The staff testing service only started at the beginning of April which coincided with the peak of the pandemic in the UK. In addition, priority was given to staff in clinical areas that were thought to be at highest risk e.g. accident and emergency and intensive care. The criteria have since changed in accordance with UK government guidance.

The majority of staff reporting symptoms consistent with possible SARS-CoV-2 infection tested negative but at a lower rate than previous reports [4,5] and allows for a strategy to maximise the available workforce. The incidence of SARS - CoV-2 RNA detection dropped significantly over the four week period reported and mirrors the pattern shown in the national testing programme [1]. Along with Hunter and colleagues [5], this supports the suggestion that most frontline healthcare workers contract the virus outside of the care setting and that remained the case as the pandemic progressed in the UK with a higher community prevalence. Furthermore, SARS -CoV-2 RNA detection is not associated with any particular age group, sex, occupation or ethnicity and no interaction was found between these variables. Importantly, although no specified characteristics were associated with contracting the virus, this study cannot comment upon whether any of these variables are risk factors for more severe disease progression [10,11].

Whilst the UK appears to be "past the peak" of the pandemic it is clear that SARS-CoV-2 will continue to influence our working lives for many months to come. Understanding the role of a staff testing programme allows for integration of this into a number of the recovery strategies for the UK including "test, track and trace". Such a service will also be crucial should there be a second peak of infection in the UK. The present strategy shows that active testing of symptomatic healthcare workers is effective for identifying positive cases to allow isolation but also to maximise the capacity of the remaining workforce. There is also some evidence that uncertainty about SARS-CoV-2 status leads to a reduction in the available workforce and therefore a robust testing strategy gives confidence to healthcare workers about returning to the working environment [4]. It could be argued that testing of all healthcare workers would be a more robust strategy, however, the policy in the UK at the time was to test symptomatic healthcare workers only. There is certainly some evidence for targeting testing for healthcare workers performing or involved in higher risk activities such as aerosol generating procedures [12]. The reliability of the test in asymptomatic individuals was uncertain as was the infectivity of asymptomatic infected individuals. This study also provides additional evidence to healthcare workers in the BAME community that they are not at increased risk of contracting SARS-CoV-2.

There are some differences and potential limitations in this study. This study was conducted during a different phase of the pandemic to the 2 previous UK reports with an increased SARS - CoV-2 RNA detection rate. This may reflect more stringent testing criteria but is more likely representative of the background population prevalence of SARSCoV-2 in the community. Most staff members tested during the first 10 - 14 days will have contracted the virus in late March when testing in our institution was not available. Data in relation to specific symptoms were not collated in this study, whether one or both symptoms are more likely to be associated with SARS-CoV-2 is unknown. One of the main issues is the sensitivity of the testing kit used for detecting SARS$\mathrm{CoV}-2$ which is quoted as $80 \%$ at best [13], but for the first three weeks our service employed routine repeat testing for those with a "not detected" result to minimise false negative results [14]. This only identified a further 16 individuals and was therefore discontinued with the assumption that our methods were yielding lower false negatives than expected. The specificity has been judged to be very high with the lowest estimate being 95\% [15]. If an additional 5\% of cases were to have been found as per the previous three weeks, this would have increased the incidence to $80 / 210$ which would still be significantly lower than the previous 3 weeks $(p=0.029)$. Repeat testing of previously positive and recovered individuals was not undertaken routinely and no staff member presented with a repeat episode of symptoms during the study period. Similarly, no asymptomatic staff were tested, as per the national strategy, but this may also have affected the overall incidence. Further limitations include some missing data particularly in the occupation and ethnicity variables although only individuals with complete datasets were entered into the multivariable analysis. Further sub-classification of occupational role would have added more insight into risk of testing positive as they are currently quite broad. For example, an outpatient nurse has a different exposure to a pathologist who has a different exposure to an ICU nurse. Other data such as underlying comorbidities were not recorded which may also have played a role. Also some variables only had very small numbers such as clinical support staff. The majority of staff tested were clinical and predominantly medical and nursing staff as would be expected and the initial testing strategy was aimed at those in areas such as intensive care and emergency services. The number of individuals with BAME coding was also small but made up a larger proportion of the testing population compared to the proportion registered in the workforce suggesting similar access to testing to their non-BAME colleagues or potentially more concern for personal risk $[8,9,16-18]$. The majority were from the Indian subcontinent but some ethnic groups were only present in very small numbers and some were not represented at all (e.g. Caribbean). This limited the analysis of the BAME group overall as much larger numbers would be required to determine if a particular ethnic group were associated with a higher risk. The prevalence of BAME healthcare workers is higher in other areas of the country and therefore this should be interpreted with caution and larger studies conducted. Again, this data cannot comment upon the clinical course of those who contracted SARS-CoV-2 and this is currently a research priority [7].

In conclusion, a robust healthcare worker testing strategy is an important component of managing the workforce during the SARSCoV-2 pandemic. No specific variables were identified that altered the risk of SARS-CoV-2 RNA detection including age, sex, occupation and ethnicity. To understand whether there are any risk factors associated with increased risk of SARS-CoV-2 a large, multi-centre study would be required.

\section{Author contributions}

JSL and CML conceived the study. JSL, VR, TJ, NS, JC and CML collected and interpreted the data. JSL performed the data analysis. JSL 
and CML wrote the manuscript. JSL, VR, TJ, NS, JC, and CML edited and approved the final manuscript.

\section{Data sharing statement}

The anonymised database can be made available from publication until 36 months after publication. Data will only be shared with individuals who have obtained appropriate approval from a recognised body with authority to access such data. Access should be via the corresponding author.

\section{Declaration of Competing Interest}

None declared for all authors. Dr Clare M Leeds affirms that the manuscript is an honest, accurate, and transparent account of the study being reported; that no important aspects of the study have been omitted; and that any discrepancies from the study as originally planned (and, if relevant, registered) have been explained.

\section{Supplementary materials}

Supplementary material associated with this article can be found, in the online version, at doi: 10.1016/j.eclinm.2020.100513.

\section{References}

[1] Public Health England (PHE). COVID-19: track coronavirus cases. London: PHE 2020 https://www.gov.uk/government/publications/covid-19-track-coronaviruscases. Accessed 4 May 2020.

[2] Zhang Z, Liu S, Xiang M, et al. Protecting healthcare personnel from 2019-nCoV infection risks: lessons and suggestions. Front Med 2020;14(2):229-31 Mar.

[3] Wu Z, McGoogan JM. Characteristics of and important lessons from the coronavirus disease 2019 (COVID-19) outbreak in China: summary of a report of 72314 cases from the chinese center for disease control and prevention. JAMA 2020;323 (13):1239-42

[4] Keeley AJ, Evans C, Colton $\mathrm{H}$, et al. Roll-out of SARS-CoV-2 testing for healthcare workers at a large NHS Foundation Trust in the United Kingdom, March 2020. Euro Surveill 2020;25(14):2000433.

[5] Hunter E, Price DA, Murphy E. First experience of COVID-19 screening of healthcare workers in England. Lancet 2020;395(10234):e77-8.

[6] Canova V, Lederer Schläpfer H, Piso RJ, et al. Transmission risk of SARS-CoV-2 to healthcare workers -observational results of a primary care hospital contact tracing. Swiss Med Wkly 2020;150:w20257.

[7] Rimmer A. Covid-19: disproportionate impact on ethnic minority healthcare workers will be explored by government. BMJ 2020;369:m1562.

[8] Khunti K, Singh AK, Pareek M, Hanif W. Is ethnicity linked to incidence or outcomes of covid-19? BMJ 2020;369:m1548.

[9] Rimmer A. Two thirds of healthcare workers who have died were from ethnic minorities. BMJ 2020;369:m1621.

[10] Hu L, Chen S, Fu Y, et al. Risk factors associated with clinical outcomes in 323 COVID-19 hospitalized patients in Wuhan, China. Clin Infect Dis 2020: ciaa539.

[11] Zhang J, Yu M, Tong S, Liu LY, Tang LV. Predictive factors for disease progression in hospitalized patients with coronavirus disease 2019 in Wuhan, China. J Clin Virol 2020;127:104392.

[12] El-Boghdadly K, Wong DJN, Owen R, et al. Risks to healthcare workers following tracheal intubation of patients with COVID-19: a prospective international multicentre cohort study. Anaesthesia 2020. doi: 10.1111/anae.15170.

[13] Chan JF, Yip CC, To KK, et al. Improved molecular diagnosis of COVID-19 by the novel, highly sensitive and specific COVID-19-RdRp/Hel real-time reverse transcription-PCR assay validated in vitro and with clinical specimens. J Clin Microbiol 2020;58(5):e00310-20

[14] Shen N, Zhu Y, Wang X, et al. Characteristics and diagnosis rate of 5,630 subjects receiving SARS-CoV-2 nucleic acid tests from Wuhan, China. JCI Insight 2020:5 (10):e137662.

[15] Watson J, Whiting PF, Brush JE. Interpreting a covid-19 test result. BMJ 2020;369: m1808. doi: $10.1136 / \mathrm{bmj} . \mathrm{m} 1808$.

[16] Siddique H. UK government urged to investigate coronavirus deaths of BAME doctors; 2020. https://www.theguardian.com/society/2020/apr/10/uk-coronavirus-deaths-bame-doctors-bma

[17] Croxford R. Coronavirus cases to be tracked by ethnicity; 2020. https://www.bbc. co.uk/news/health-52338101

[18] Cook T, Kursumovic E, Lennane S. Exclusive: deaths of NHS staff from covid-19 analysed. Health Sci J 2020. https://www.hsj.co.uk/exclusive-deaths-of-nhs-stafffrom-covid-19-analysed/7027471.article. 